\title{
Genetic Analysis of CHST6 Gene in Indian Families with Macular Corneal Dystrophy
}

\author{
Durga Murugan' ${ }^{1}$ Namperumalsamy Venkatesh Prajna ${ }^{2}$, Lumbini Devi², Periasamy \\ Sundaresan ${ }^{1 *}$ \\ ${ }^{1}$ Department of Molecular Genetics, Aravind Medical Research Foundation, Aravind Eye Hospital, Madurai, Tamilnadu, \\ India \\ ${ }^{2}$ Cornea Clinic, Aravind Eye Hospital, Madurai, Tamilnadu, India
}

Received: November 22, 2017; Accepted: November 30, 2017; Published: December 06, 2017

*Corresponding author: Periasamy Sundaresan, Senior Scientist-V, Department of Genetics, Aravind Medical Research Foundation, Aravind Eye Hospital, Tamil Nadu, India, Tel: 91452 4356100; Fax: 91452 2530984; E-mail: sundar@aravind.org

\begin{abstract}
Background:Unlike the western world, Macular Corneal Dystrophy (MCD) is the most common corneal stromal dystrophy in India. It is caused by mutations in the carbohydrate sulfotransferase 6 (CHST6) gene. So far, there are limited numbers of reports on CHST6 screening. Therefore, our screening of CHST6 gene in Indian families with MCD will be useful for genetic diagnosis, carrier detection and genetic counseling to families included in this study and other families with similar disease condition.
\end{abstract}

Purpose: The main purpose of this study was to perform the genetic analysis of 55 Indian MCD families.

Methods: We have recruited 55 affected, 11 unaffected members from 55 MCD families along with 100 controls. All the study subjects underwent ocular examination before collecting the blood for the screening of CHST6 gene. Polymerase chain reaction was performed followed by bi-directional sequencing. The novel mutations were predicted by Polyphene-2, SIFT, Mutation taster and SOPMA tool.

Results: We identified 14 different mutations, 3 known SNP's in 44 MCD patients amongst 6 were novel. Also 2 hotspot mutations were identified amongst the 14 mutations. We could not identify any mutations in the coding region of CHST6 gene in 11 MCD patients (20\%).

Conclusion: Our study identified six novel mutations which will add up to the list of already known mutations identified in different ethnic populations. Our study also increases the mutational landscape of CHST6 gene. We concluded due to genetic heterogeneity there might be some other gene involved in Indian MCD patients who are negative for CHST6 mutations.

Keywords: CHST6 gene; Cornea; Heterogeneity; Mutations;

\section{Abbreviations}

MCD: Macular Corneal Dystrophy; CHST6: Carbohydrate Sulfotransferase 6; SIFT: Sorting Intolerant From Tolerant; SOPMA: Self-Optimized Prediction Method with Alignment;

\section{Introduction}

Macular corneal dystrophy is an autosomal recessive disorder, caused by CHST6 gene [1]. It was first described by Groenouw in 1890. Due to high degree of consanguinity in some ethnic population, this disorder is highly prevalent in Saudi Arabia and South Indian population [2,3]. The Clinical manifestations of MCD usually occurs by irregular, focal haze formation that leads to greywhite opacities eventually leading to decreased visual acuity by the fifth decade of life [4]. Slit lamp view of cornea of patient with MCD showing diffusely distributed, rounded stromal opacities (Figure 1). In early stages, Photo Therapeutic Keratectomy (PTK) can be done, whereas in advanced disease stage, it requires full thickness or deep anterior lamellar keratoplasty $[5,6]$. The recurrence rate of corneal opacities is more than $40 \%$ in macular corneal grafts even after 10 years of Penetrating Keratoplasty (PKP) [7].

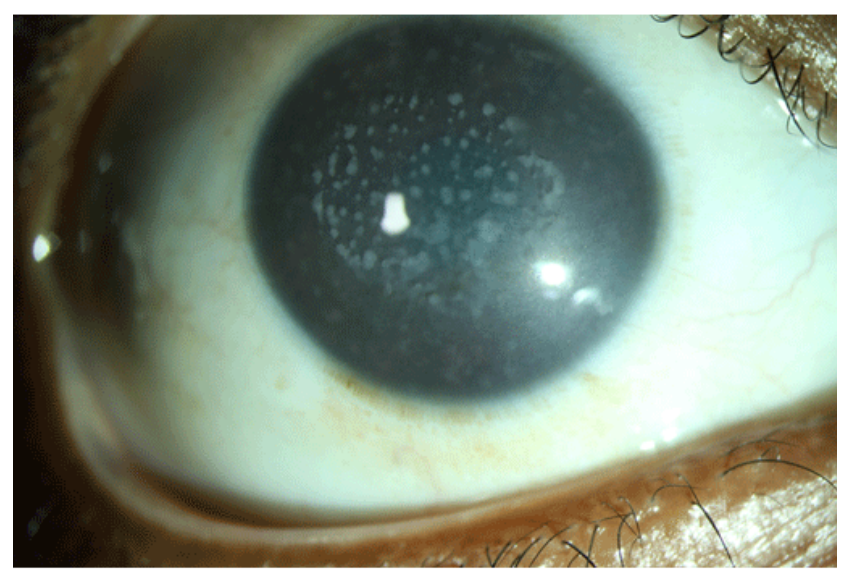

Figure 1: Slit lamp view of cornea with macular dystrophy demonstrating multiple irregular grey-white opacities with intervening central stromal haze. 
CHST6 gene is the only candidate gene so far known in MCD and has been further screened in different ethnic populations across the world [2,3,8-17]. It is located on chromosome $16 q 22$ $[18,19]$. It encodes a golgi resident enzyme $\mathrm{N}$-acetyl glucosamine6-0-sulfotransferase (C-GlcNac-6-ST) that catalyses the sulfation of keratan sulfate (KS) essential for corneal transparency [2022]. Defect in CHST6 gene results in unsulfated keratan sulfate deposition eventually leading to MCD phenotype [23].

Based on the histochemical features, MCD is classified into three immunophenotypes I, II and IA [24-26]. Type I is characterized by absence or low level of sulfated KS (AgKS) in cornea as well as serum. Type II is characterized by normal or marginally reduced level of AgKS in cornea as well as serum. Type IA is characterized by low level of antigenic AgKS in serum and detectable level in keratocytes [26].

The main purpose of this study was to screen the coding region of CHST6 gene in 55 Indian families with MCD.

\section{Materials and Methods}

\section{Patients}

The subjects for this study (cases and controls) were recruited from the outpatient services of a tertiary eye care hospital in South India (Aravind Eye Care System, Madurai, India). The study adhered to the tenets of the Declaration of Helsinki, and ethics committee approval was obtained from the Institutional Ethics Committee of the Aravind Eye Care System. All subjects read and signed informed consent except for illiterate subjects, who had the information leaflet read out and provided a thumb impression for participation consent.

\section{Genetic Analysis}

\section{DNA extraction and PCR amplification}

With informed consent from the study subjects, $5 \mathrm{ml}$ of blood was collected from all the study subjects and genomic DNA was extracted by salting out method [27]. There are four coding regions in CHST6 gene [18,19]. Out of these, exon 3 is unique as already reported in several independent studies. Based on the uniqueness of exon 3, we have also targeted the exon 3 by using predesigned primers [16]. Each PCR was carried out in a $50 \mu \mathrm{l}$ reaction mixture containing 100 ng of genomic DNA, $1 \mathrm{X}$ buffer (PCR buffer (10 mM TRIS hydrochloride, pH 8.3; $50 \mathrm{mM}$ potassium chloride; $1.5 \mathrm{mM}$ magnesium chloride and $0.001 \%$ gelatin)), 0.5 pmol of each primer $200 \mu \mathrm{M}$ of deoxynucleotide triphosphate and $1 \mathrm{U}$ of Taq DNA polymerase (Sigma Aldrich). Amplification was performed in a DNA Thermal cycler (Applied Biosystems-Invitrogen). The thermal cycling program started with an initial denaturation of 10 minutes at $96^{\circ} \mathrm{C}$, followed by 37 cycles of $96^{\circ} \mathrm{C}$ for 30 seconds, $60^{\circ} \mathrm{C}$ for 30 seconds of annealing, $72^{\circ} \mathrm{C}$ for 45 seconds with a final extension at $72^{\circ} \mathrm{C}$ for 5 minutes

\section{Sanger Sequencing}

The amplified DNA products were purified by QIA quick PCR purification kit method (Bio Basic Inc.)) followed by cyclic PCR. Bi-directional sequencing was performed (3130 Genetic
Analyser; Applied Biosystems) and the results were compared with the reference sequences of CHST6 gene using BLAST and Chromas lite (2.1) software.

\section{Bioinformatics Analysis}

The Self-Optimized Prediction Method with Alignment (SOPMA) tool (https://npsa prabi.ibcp.fr/NPSA/npsa_sopma. html) was used for the prediction of secondary structure of CHST6 protein. In addition, Polyphene 2, SIFT and Mutation taster were used for predicting the pathogenicity of novel mutations identified in CHST6 gene and also the conservation of the novel mutations identified was being analysed by CLUSTALW (1.2.2) multiple sequence alignment software (http://www.clustal.org/ omega).

\section{Results}

We have recruited 55 families with MCD at the cornea unit of Aravind eye care system. Out of the 55 families, 30 families were carrying history of consanguineous marriages. All the study subjects were screened for CHST6 gene mutations. Interestingly, we identified 6 novel mutations (Ser53X, Ser81X, Val172Met, Arg202His, Ser248Asp, Glu274Gln) in 7 different MCD families and 8 known mutations (H42Y, S53L, R93H, R127C, Q58X, V66VfsX3, Q182RfsX198, N194_196delinsRC) in 29 different MCD families. Also we have identified 3 known single nucleotide polymorphisms (R50C, R205Q, R205W) in 8 families described in Table 1 . Rest of the unaffected 11 family members was negative for these mutations. In addition, 100 age matched controls were also negative for these mutations. We considered 6 novel sequence variants as mutations based on the following criteria:

1. The six novel changes were segregating in the family in an autosomal recessive fashion.

2. All the six novel mutations were absent in the following databases (Ensemble, HGVS, 1000 genome project)

3. Three of the novel missense mutations were highly conserved (Val172Met, Ser248Asn, Glu274Gln) and one novel missense mutation was not conserved (Arg202His) across different orthologous species (Figure 2)

4. Mutation taster predicted all the novel mutations to be disease causing and the polyphen2, SIFT programme predicted all are damaging.

A novel homozygous missense mutation Val172Met was identified in a patient from family 5 with consanguinity, Arg202His was identified in a patient from family 42 with consanguinity, Ser248Asn was present in a patient from family 25 with consanguinity, Glu274Gln was identified in a patient from family 9 with consanguinity (Table 1). Additionally, a novel homozygous nonsense mutation Ser53X was identified in 2 patients (patient \& patient's younger brother) from family 8 without consanguinity but with MCD history (Figure 3a). One more novel homozygous nonsense mutation Ser81X was identified in a patient from family 36 without consanguinity and MCD history. 


\begin{tabular}{|c|c|c|c|c|c|c|}
\hline $\begin{array}{c}\text { Family/ } \\
\text { Patient number }\end{array}$ & Mutation $^{\mathrm{a}}$ & Mutation type & $\begin{array}{c}\text { Amino acid } \\
\text { R-group change }\end{array}$ & $\begin{array}{l}\text { Age(yrs)/ } \\
\text { Sex }\end{array}$ & & $\begin{array}{c}\text { Novel/ Previously } \\
\text { reported }^{\mathbf{b}}\end{array}$ \\
\hline 1 & No mutation & & & $32 / \mathrm{M}$ & No & \\
\hline 2 & $\begin{array}{l}\text { c.614G }>A \text { (p.R205Q) } \\
\text { dbSNP:rs377706989 }\end{array}$ & $\begin{array}{l}\text { Homozygous } \\
\text { missense }\end{array}$ & Basic to polar & $35 / \mathrm{M}$ & No & India \\
\hline 3 & c.172C>T (p.Q58X) & $\begin{array}{l}\text { Homozygous } \\
\text { nonsense }\end{array}$ & Basic to Polar & $44 / \mathrm{M}$ & Yes & France \\
\hline 4 & No mutation & & & $26 / \mathrm{M}$ & No & \\
\hline 5 & c.514G>A (p.V172M) & $\begin{array}{l}\text { Homozygous } \\
\text { missense }\end{array}$ & $\begin{array}{l}\text { Non-polar to non- } \\
\text { polar }\end{array}$ & $44 / \mathrm{M}$ & Yes & Novel \\
\hline 6 & c.198delC (p.V66VfsX3) & $\begin{array}{c}\text { Heterozygous } \\
\text { deletion }\end{array}$ & $\begin{array}{l}\text { Non-polar to non- } \\
\text { polar }\end{array}$ & $18 / \mathrm{M}$ & No & India \\
\hline 7 & No mutation & & & $10 / \mathrm{M}$ & Yes & \\
\hline 8 & c.158C>A (p.S53X) & $\begin{array}{l}\text { Homozygous } \\
\text { nonsense }\end{array}$ & Polar to stop codon & $30 / \mathrm{M}$ & No & Novel \\
\hline 9 & c.820G>C (p.E274Q) & $\begin{array}{l}\text { Homozygous } \\
\text { missense }\end{array}$ & Acidic to polar & $60 / \mathrm{M}$ & Yes & Novel \\
\hline 10 & No mutation & & & $66 / \mathrm{M}$ & No & \\
\hline 11 & $\begin{array}{l}\text { c.614G>A (p.R205Q) } \\
\text { dbSNP:rs377706989 }\end{array}$ & $\begin{array}{l}\text { Heterozygous } \\
\text { missense }\end{array}$ & Basic to polar & $23 / \mathrm{M}$ & No & India \\
\hline 12 & c.158C>T (p.S53L) & $\begin{array}{l}\text { Homozygous } \\
\text { missense }\end{array}$ & Polar to basic & $32 / \mathrm{M}$ & Yes & $\begin{array}{l}\text { Hotspot mutation } \\
\text { India, America }\end{array}$ \\
\hline 13 & No mutation & & & $50 / \mathrm{F}$ & Yes & \\
\hline 14 & c.545delA (p.Q182RfsX198) & $\begin{array}{c}\text { Heterozygous } \\
\text { deletion }\end{array}$ & Polar to basic & $47 / F$ & Yes & $\begin{array}{l}\text { Hotspot mutation } \\
\text { India }\end{array}$ \\
\hline 15 & c.158C>T (p.S53L) & $\begin{array}{l}\text { Homozygous } \\
\text { missense }\end{array}$ & Polar to non-polar & $16 / F$ & Yes & $\begin{array}{l}\text { Hotspot mutation } \\
\text { India, America }\end{array}$ \\
\hline 16 & c.158C>T (p.S53L) & $\begin{array}{l}\text { Homozygous } \\
\text { missense }\end{array}$ & Polar to non-polar & $32 / \mathrm{M}$ & Yes & $\begin{array}{l}\text { Hotspot mutation } \\
\text { India, America }\end{array}$ \\
\hline 17 & c.379C>T (p.R127C) & $\begin{array}{l}\text { Homozygous } \\
\text { missense }\end{array}$ & Basic to polar & $33 / \mathrm{M}$ & No & Saudi Arabia, India \\
\hline 18 & No mutation & & & $20 / \mathrm{M}$ & Yes & \\
\hline 19 & No mutation & & & $38 / \mathrm{F}$ & Yes & \\
\hline 20 & c.545delA (p.Q182RfsX198) & $\begin{array}{c}\text { Homozygous } \\
\text { deletion }\end{array}$ & Polar to basic & $28 / \mathrm{F}$ & Yes & $\begin{array}{l}\text { Hotspot mutation } \\
\text { India }\end{array}$ \\
\hline 21 & No mutation & & & $26 / \mathrm{F}$ & No & \\
\hline 22 & c.545delA (p.Q182RfsX198) & $\begin{array}{l}\text { Homozygous } \\
\text { deletion }\end{array}$ & Polar to basic & $27 / F$ & No & $\begin{array}{l}\text { Hotspot mutation } \\
\text { India }\end{array}$ \\
\hline 23 & No mutation & & & $28 / \mathrm{F}$ & Yes & \\
\hline 24 & c.545delA (p.Q182RfsX198) & $\begin{array}{c}\text { Homozygous } \\
\text { deletion }\end{array}$ & Polar to basic & $19 / \mathrm{F}$ & Yes & $\begin{array}{l}\text { Hotspot mutation } \\
\text { India }\end{array}$ \\
\hline 25 & c.743G>A (p.S248N) & $\begin{array}{l}\text { Homozygous } \\
\text { missense }\end{array}$ & Polar to Polar & $37 / \mathrm{M}$ & Yes & Novel \\
\hline 26 & c.614G>A (p.R205Q) & $\begin{array}{l}\text { Homozygous } \\
\text { missense }\end{array}$ & Basic to polar & $12 / \mathrm{M}$ & No & India \\
\hline
\end{tabular}

Citation: Sundaresan P, Durga M, Namperumalsamy VP, Devi L (2017) Genetic Analysis of CHST6 Gene in Indian Families with Macular Corneal Dystrophy. Int J Gen Sci 4(1): 1-10. 


\begin{tabular}{|c|c|c|c|c|c|c|}
\hline 27 & c.124C>T (p.H42Y) & $\begin{array}{l}\text { Homozygous } \\
\text { missense }\end{array}$ & Basic to polar & $30 / F$ & No & India \\
\hline 28 & c.820G >C (p.E274Q) & $\begin{array}{l}\text { Heterozygous } \\
\text { missense }\end{array}$ & Acidic to polar & $47 / F$ & Yes & India \\
\hline 29 & $\begin{array}{l}\text { c.148C>T (p.R50C) } \\
\text { dbSNP:rs28937877 }\end{array}$ & $\begin{array}{c}\text { Homozygous } \\
\text { missense }\end{array}$ & Basic to polar & $40 / \mathrm{M}$ & No & India \\
\hline 31 & $\begin{array}{l}\text { c.614G>A (p.R205Q) } \\
\text { dbSNP:rs377706989 }\end{array}$ & $\begin{array}{l}\text { Homozygous } \\
\text { missense }\end{array}$ & Basic to polar & $52 / \mathrm{F}$ & No & India \\
\hline 32 & No mutation & & & $52 / \mathrm{F}$ & Yes & \\
\hline 33 & c.158C>T (p.S53L) & $\begin{array}{l}\text { Homozygous } \\
\text { missense }\end{array}$ & Polar to non-polar & $20 / \mathrm{M}$ & No & $\begin{array}{c}\text { Hotspot mutation } \\
\text { India, America }\end{array}$ \\
\hline 34 & c.158C>T (p.S53L) & $\begin{array}{l}\text { Homozygous } \\
\text { missense }\end{array}$ & Polar to non-polar & $35 / F$ & Yes & $\begin{array}{l}\text { Hotspot mutation } \\
\text { India, America }\end{array}$ \\
\hline 35 & c.545delA (p.Q182RfsX198) & $\begin{array}{c}\text { Heterozygous } \\
\text { deletion }\end{array}$ & Polar to Basic & $50 / \mathrm{M}$ & No & $\begin{array}{c}\text { Hotspot mutation } \\
\text { India }\end{array}$ \\
\hline 36 & c.242C>A (p.S81X) & $\begin{array}{c}\text { Homozygous } \\
\text { nonsense }\end{array}$ & Polar to stop codon & $33 / \mathrm{M}$ & No & Novel \\
\hline 37 & $\begin{array}{l}\text { c.613C > T (p.R205W) } \\
\text { dbSNP:rs750219546 }\end{array}$ & $\begin{array}{l}\text { Homozygous } \\
\text { missense }\end{array}$ & Basic to non-polar & $46 / \mathrm{M}$ & No & Korea \\
\hline 38 & c.545delA (p.Q182RfsX198) & $\begin{array}{c}\text { Homozygous } \\
\text { deletion }\end{array}$ & Polar to basic & $30 / \mathrm{F}$ & Yes & $\begin{array}{c}\text { Hotspot mutation } \\
\text { India }\end{array}$ \\
\hline 39 & c.545delA (p.Q182RfsX198) & $\begin{array}{c}\text { Homozygous } \\
\text { deletion }\end{array}$ & Polar to basic & $22 / \mathrm{M}$ & Yes & $\begin{array}{c}\text { Hotspot mutation } \\
\text { India }\end{array}$ \\
\hline 40 & c.158C>T (p.S53L) & $\begin{array}{c}\text { Homozygous } \\
\text { missense }\end{array}$ & Polar to non-polar & $30 / \mathrm{M}$ & Yes & $\begin{array}{l}\text { Hotspot mutation } \\
\text { India, America }\end{array}$ \\
\hline 41 & $\begin{array}{l}\text { c.148C>T (p.R50C) } \\
\text { dbSNP:rs28937877 }\end{array}$ & $\begin{array}{l}\text { Homozygous } \\
\text { missense }\end{array}$ & Basic to polar & $25 / F$ & No & India \\
\hline 42 & c.290G >A (p.R202H) & $\begin{array}{l}\text { Homozygous } \\
\text { missense }\end{array}$ & Basic to basic & $21 / \mathrm{M}$ & Yes & Novel \\
\hline 43 & c.545delA (p.Q182RfsX198) & $\begin{array}{c}\text { Homozygous } \\
\text { deletion }\end{array}$ & Polar to basic & $36 / \mathrm{M}$ & No & $\begin{array}{c}\text { Hotspot mutation } \\
\text { India }\end{array}$ \\
\hline 44 & c.545delA (p.Q182RfsX198) & $\begin{array}{c}\text { Heterozygous } \\
\text { deletion }\end{array}$ & Polar to basic & $61 / \mathrm{M}$ & No & $\begin{array}{c}\text { Hotspot mutation } \\
\text { India }\end{array}$ \\
\hline 45 & c.158C>T (p.S53L) & $\begin{array}{l}\text { Homozygous } \\
\text { missense }\end{array}$ & Polar to non-polar & $25 / M$ & Yes & $\begin{array}{c}\text { Hotspot mutation } \\
\text { India, America }\end{array}$ \\
\hline 46 & c.545delA (p.Q182RfsX198) & $\begin{array}{c}\text { Heterozygous } \\
\text { deletion }\end{array}$ & Polar to basic & $60 / \mathrm{M}$ & No & $\begin{array}{c}\text { Hotspot mutation } \\
\text { India }\end{array}$ \\
\hline 47 & c.545delA (p.Q182RfsX198) & $\begin{array}{c}\text { Homozygous } \\
\text { deletion }\end{array}$ & Polar to basic & $27 / F$ & Yes & $\begin{array}{c}\text { Hotspot mutation } \\
\text { India }\end{array}$ \\
\hline 48 & c.158C>T (p.S53L) & $\begin{array}{l}\text { Homozygous } \\
\text { missense }\end{array}$ & Polar to non-polar & $38 / F$ & Yes & $\begin{array}{c}\text { Hotspot mutation } \\
\text { India, America }\end{array}$ \\
\hline 49 & c.158C>T (p.S53L) & $\begin{array}{l}\text { Homozygous } \\
\text { missense }\end{array}$ & Polar to non-polar & $31 / \mathrm{M}$ & Yes & $\begin{array}{c}\text { Hotspot mutation } \\
\text { India, America }\end{array}$ \\
\hline
\end{tabular}




\begin{tabular}{|c|c|c|c|c|c|c|}
\hline 50 & c.158C>T (p.S53L) & $\begin{array}{l}\text { Homozygous } \\
\text { missense }\end{array}$ & Polar to non-polar & $17 / F$ & No & $\begin{array}{l}\text { Hotspot mutation } \\
\text { India, America }\end{array}$ \\
\hline 51 & $\begin{array}{l}\text { c.581_586 delACCTACinsGGT } \\
\text { (p.N194_R196delinsRC) }\end{array}$ & $\begin{array}{l}\text { Deletioninsertion } \\
\text { mutation }\end{array}$ & Non-polar to polar & $39 / \mathrm{M}$ & Yes & India \\
\hline 52 & No mutation & & & $36 / F$ & Yes & India \\
\hline 53 & c.278G>A (p.R93H) & $\begin{array}{l}\text { Homozygous } \\
\text { missense }\end{array}$ & Basic to Basic & $40 / \mathrm{M}$ & Yes & India \\
\hline 54 & $\begin{array}{l}\text { c.148C > T (p.R50C) } \\
\text { dbSNP:rs28937877 }\end{array}$ & $\begin{array}{l}\text { Homozygous } \\
\text { missense }\end{array}$ & Basic to polar & $37 / F$ & Yes & India \\
\hline 55 & $\begin{array}{l}\text { c.581_586 delACCTACinsGGT } \\
\text { (p.N194_R196delinsRC) }\end{array}$ & $\begin{array}{l}\text { Deletioninsertion } \\
\text { mutation }\end{array}$ & Non-polar to polar & $44 / \mathrm{M}$ & No & India \\
\hline
\end{tabular}

Table 1. Details of CHST6 gene mutations identified in 55 MCD patients. Mutationa represents the nomenclature of mutations according to the current recommendations in http://www.hgvs.org/mutnomen/recs.html\#DNA. Reportedb represents the novel or previously reported mutations in the specific population. dbSNP represents single nucleotide polymorphism.

\begin{tabular}{|c|c|}
\hline & S53X \\
\hline CHST6_HUMAN & LSSWRSGS--.-- \\
\hline CHST6_PANTR & LSSWRSASFFVGQLFSQ \\
\hline CHST6_MOUSE & LSSWRSASSFVGQLFSQ \\
\hline CHST6_BOVIN & LSSWRSQSFFVGQLFSQ \\
\hline \multirow[t]{2}{*}{ CHST6_GORGO } & LSSWRSQSFFGQLFSQ \\
\hline & $\begin{array}{r}\text { S81X } \\
\text {. }\end{array}$ \\
\hline CHST6_HUMAN & MEPAWHVWTTIS--- \\
\hline CHST6_PANTR & MEPAWHVWTTISQGSAA \\
\hline CHST6_MOUSE & MEPAWHVWDT QGSAP \\
\hline CHST6_BOVIN & MEPAWHVWAAI SQGSAL \\
\hline \multirow[t]{2}{*}{ CHST6_GORGO } & MEPAWHVWTTISQGSAA \\
\hline & $\begin{array}{c}\text { V172M } \\
\text { EACRSYSHYMLEVRFF }\end{array}$ \\
\hline CHST6_PANTR & EACRSYSHYVLKEVRFF \\
\hline CHST6_MOUSE & EACSSYSHVVKKEVRFF \\
\hline CHST6_BOVIN & EACRSYSHVYLKEVRFF \\
\hline \multirow[t]{2}{*}{ CHST6_GORGO } & EACRSYSHVULKEVRFF \\
\hline & R202H \\
\hline CHST6_HUMAN & YPLLSDPALNLRVHLVRD \\
\hline CHST6_PANTR & YPLLSDPALNIRIVHLVRD \\
\hline CHST6_MOUSE & YPLLSDPALNI RIVHLVRD \\
\hline CHST6_BOVIN & YPLLSDPALHI RVHLVRD \\
\hline \multirow[t]{2}{*}{ CHST6_GORGO } & YPLLSDPALNLRVHLVRD \\
\hline & $S 248 \mathrm{~N}$ \\
\hline CHST6_HUMAN & RVVREVCRSSIVRIAEAATL \\
\hline CHST6_PANTR & RVVREVCRSSVRIAEAATL \\
\hline CHST6 MOUSE & RVVNEVCFSHVRIAEAALH \\
\hline CHST6_BOVIN & RVVREVCRSSHVIAEAATR \\
\hline \multirow[t]{2}{*}{ CHST6_GORGO } & RVVREVCRSSVRIAEAATL \\
\hline & E274Q \\
\hline CHST6_HUMAN & FLRGRYRLVRFEDLAREPL \\
\hline CHST6_PANTR & FLRGRYRLVRHEDLAREPL \\
\hline CHST6_MOUSE & FLQDRYRLVRYEDLARDPL \\
\hline CHST6_BOVIN & ALRGRYRLVRPEDLARAPL \\
\hline \multirow[t]{2}{*}{ CHST6_GORGO } & FLRGRYRLVRIEDLAREPL \\
\hline & S53L \\
\hline CHST6_HUMAN & VLSSWRSGSFFVGL \\
\hline CHST6_PANTR & VLSSWRSGSFFVGQL \\
\hline CHST6 MOUSE & VLSSWRSGS\$FVGQL \\
\hline CHST6_BOVIN & VLSSWRSGS\$FVGQL \\
\hline CHST6_GORGO & VLSSWRSGSSFVGQL \\
\hline
\end{tabular}

Figure 2: Protein sequence alignment showing the conservation of amino acids in CHST6 among related mammalian species. Boxes indicate the position of mutated residue in orthologs.
In addition, we have identified two hotspot mutations in the coding region of CHST6 gene i.e. a missense mutation serine-53 to leucine (Ser53Leu) was identified in 10 patients from 10 different families (Figure $3 \mathrm{~b}$ ). We also observed a homozygous single base pair deletion (Glu182ArgfsX198) in 7 patients from 7 different families and heterozygous deletion in 4 patients from 4 different families (Figure 3c). This deletion mutation resulted in frame shift at glutamine-182 with termination after 198 amino acids leading to altered reading frame.

A heterozygous deletion (Q182RfsX198-deletion) mutation was identified in a patient from family 44. Further, pedigree analysis revealed that the propand's father, propand's elder sister and propand's elder brother had the same eye problem (No genomic DNA for analysis). But propand's two sons are phenotypically and genotypically normal. We believe, either it could be uniparentaldisomy or the second mutation may be present in the deep intronic region or regulatory region which was not covered by Sanger sequencing.

Further, a known heterozygous single base pair deletion was also identified in one of the patient from family 6 causing a frame shift at valine-66 (Val66ValfsX3) resulting in a premature termination codon at amino acid residue 2 . The second mutation in the patient could be either present in deep intronic or regulatory region of CHST6 gene which was not covered by Sanger sequencing. Furthermore, in family 3, we have identified a homozygous nonsense mutation (Glu58X) in a patient and his affected sibling that leads to the formation of truncated protein (Table 1). Interestingly, we have also identified known 6 bp deletion (homozygous state) and 3 bp Insertion (Asn194_ Arg196delinsArgCys) in two patients belonging to two unrelated families (family 51 and 55) that results in frame shifting at asparagine-194 position (Table 1). 


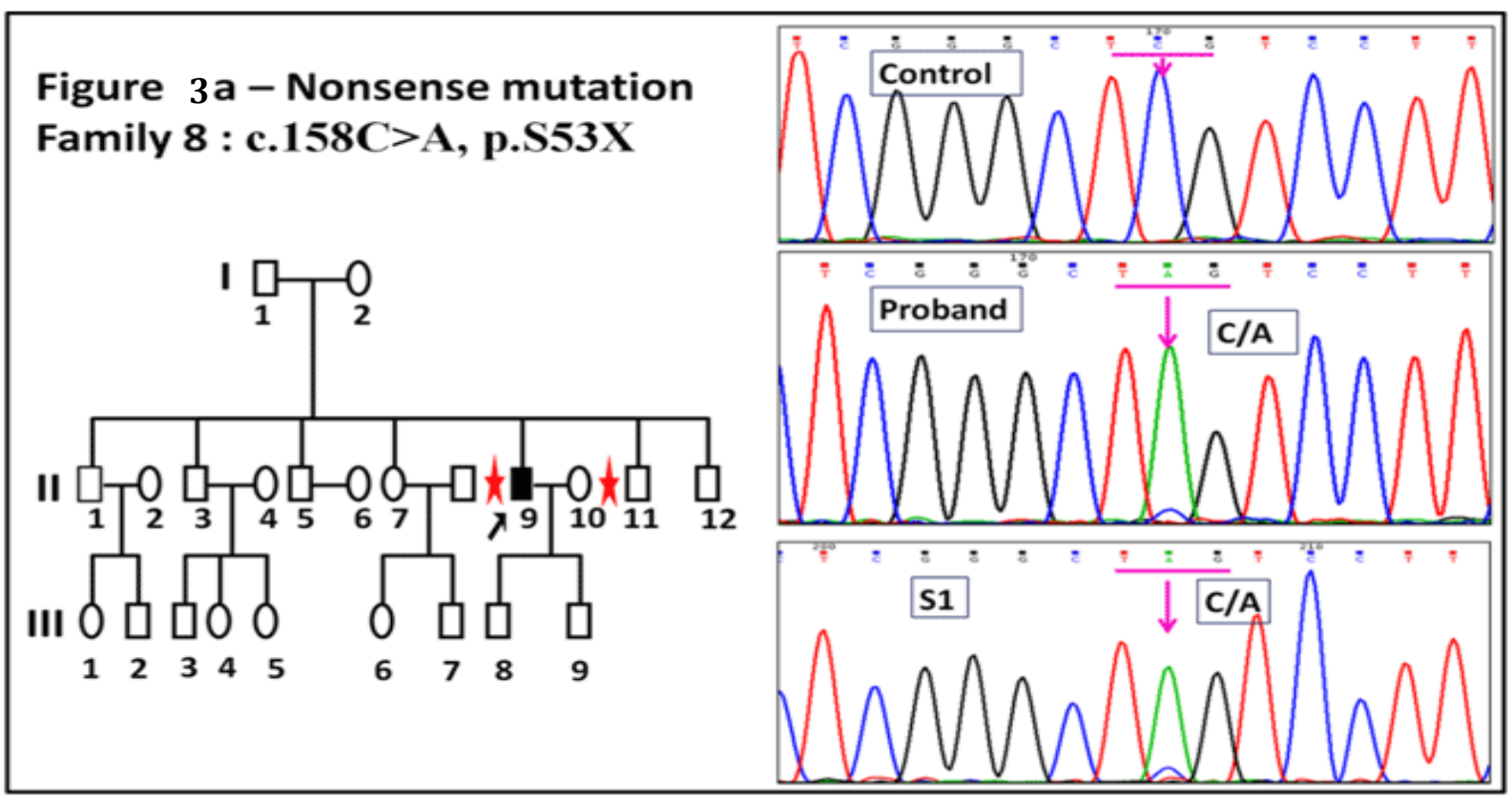

Figure 3a: Mutation analysis of CHST6 using bi-directional sequencing. I,II,III indicate generation 1,2,3; squares indicate males; circles indicate females; solid square and arrow indicate affected individual; open squares and circles indicate unaffected individuals; parallels indicate consanguinity; asterisk* indicate available sample for analysis; in the chromatogram arrow indicate codon subjected to change, and S1 indicate sibling 1.

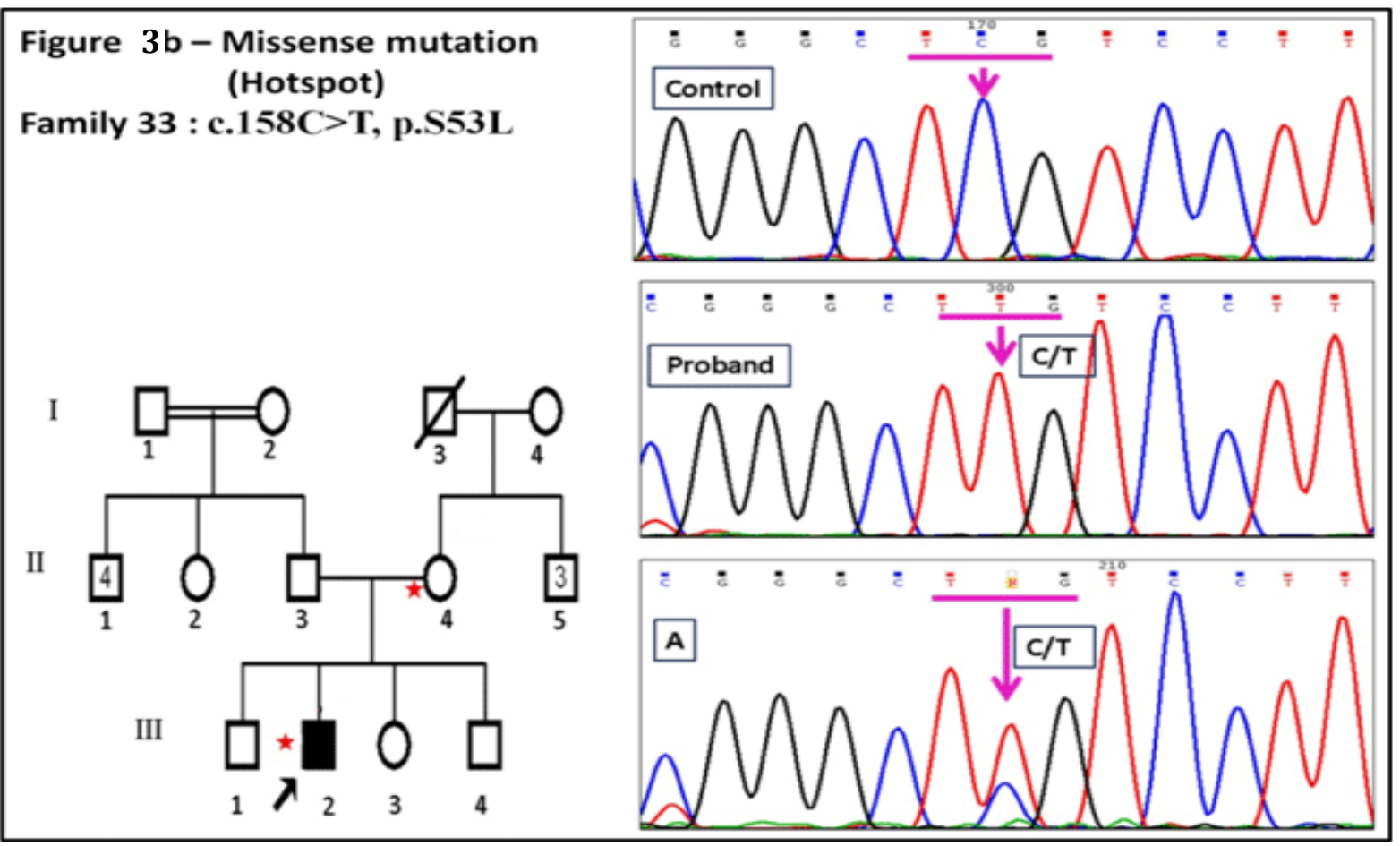

Figure 3b: I,II,III indicate generation 1,2,3; squares indicate males; circles indicate females; solid square and arrow indicate affected individual; open squares and circles indicate unaffected individuals; parallels indicate consanguinity; a slash through a square or circle indicate deceased individuals; asterisk* indicate available sample for analysis; in the chromatogram arrow indicate codon subjected to change, and A indicate propand's mother. 


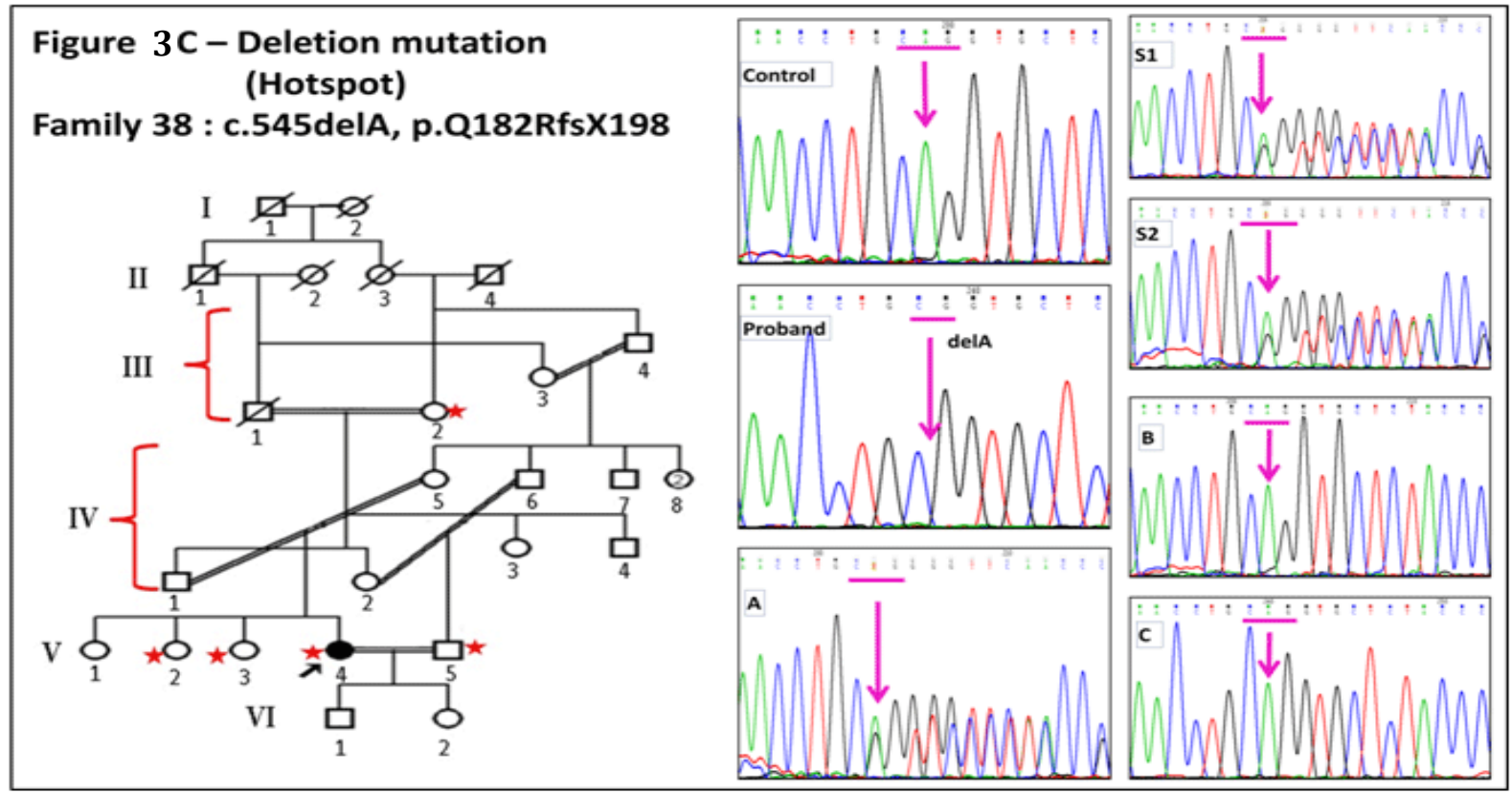

Figure 3c: I,II,III,IV,V,VI indicate generation 1,2,3,4,5,6; squares indicate males; circles indicate females; solid circle and arrow indicate affected individual; asterisk* indicate available sample for analysis; open squares and circles indicate unaffected individuals; parallels indicate consanguinity; a slash through a square or circle indicate deceased individuals; in the chromatogram arrow indicate codon subjected to change, S1,S2 indicate sibling 1,2; A indicate propand's mother; B indicate propand's husband; C indicate propand's grandmother.

Moreover, we have identified 3 known single nucleotide polymorphisms (SNP's) (rs28937877, rs377706989 and rs750219546) in eight different patients (Table 1). Of these, rs750219546 has already been identified in the ethnic cohort of Korean population providing additional support for their study.

Overall, we have sequenced 55 families, out of these families; we failed to identify any mutation in 11 MCD patients. Amongst these, 2 patients from two unrelated families had consanguinity $(7,13)$ in their family, but had no mutations in the entire coding region of CHST6 gene.

\section{Discussion}

In our current study, we have analysed 55 MCD patients including their 11 unaffected family members and 100 controls from the same ethnic background for the screening of CHST6 gene. Interestingly, we have identified 6 novel mutations in six MCD patients and 11 different known mutations in 38 patients. Previous studies have shown in Saudi Arabian and South Indian population that the prevalence of MCD is high; most probably due to the higher frequency of consanguineous marriage in these ethnic population [2,3]. In our current study, out of 55 families studied, we have also analysed 30 south Indian families with consanguineous marriage.

\begin{tabular}{|c|c|c|c|c|c|c|c|c|c|c|c|c|c|c|c|c|}
\hline \multirow[t]{2}{*}{$\begin{array}{c}\text { State of } \\
\text { amino acid }\end{array}$} & \multicolumn{2}{|c|}{ Control } & \multicolumn{2}{|c|}{$\begin{array}{l}\text { Nonsense } \\
\text { p.S53X }\end{array}$} & \multicolumn{2}{|c|}{$\begin{array}{c}\text { Nonsense } \\
\text { p.S81X }\end{array}$} & \multicolumn{2}{|c|}{$\begin{array}{l}\text { Missense } \\
\text { p.V172M }\end{array}$} & \multicolumn{2}{|c|}{$\begin{array}{l}\text { Missense } \\
\text { p.R202H }\end{array}$} & \multicolumn{2}{|c|}{$\begin{array}{c}\text { Missense } \\
\text { p.S248N }\end{array}$} & \multicolumn{2}{|c|}{$\begin{array}{c}\text { Missense } \\
\text { p.E274Q }\end{array}$} & \multicolumn{2}{|c|}{$\begin{array}{c}\text { Hotspot-missense } \\
\text { p.S53L }\end{array}$} \\
\hline & & $\%$ & & $\%$ & & $\%$ & & $\%$ & & $\%$ & & $\%$ & & $\%$ & & $\%$ \\
\hline Alpha helix & 190 & 48.1 & 15 & 28.85 & 28 & 35 & 194 & 49.11 & 190 & 48.1 & 184 & 46.58 & 190 & 46.1 & 191 & 48.35 \\
\hline Extended strand & 58 & 14.68 & 14 & 26.92 & 20 & 25 & 54 & 13.67 & 58 & 14.68 & 50 & 12.66 & 58 & 14.68 & 58 & 14.68 \\
\hline$\beta$ turn & 31 & 7.85 & 3 & 5.77 & 8 & 10 & 31 & 7.85 & 31 & 7.85 & 0 & 0 & 31 & 7 & 32 & 8.1 \\
\hline Random coil & 116 & 29.37 & 20 & 38.46 & 24 & 30 & 116 & 29.37 & 116 & 29.37 & 161 & 40.76 & 116 & 30.37 & 116 & 28.86 \\
\hline $\begin{array}{l}\text { Instability index } \\
\text { (II) }\end{array}$ & US & 46.24 & US & 58.93 & US & 50.87 & US & 46.24 & US & 46.24 & US & 45.13 & US & 45.13 & US & 45.1 \\
\hline
\end{tabular}


CHST6 gene encodes an enzyme C-GlcNac-6-ST which utilizes a sulfate group donor 3'-phospho-5'-adenylyl sulfate (PAPS) to catalyse the transfer of sulfate group to position 6 of $\mathrm{N}$-acetylglucosamine (GlcNac) residues of keratan sulfate [28]. Keratan sulfate plays a central role in the developing cornea for the acquisition of transparency and in the adult cornea for the maintenance of transparency [11,29]. The keratan sulfate is impaired in MCD cornea resulting corneal cloudiness leads to impaired vision.

The identified known mutations have been observed among patients from several populations included India Saudi Arabia, Korea, Egypt, Japan, America and France [2,3,1517,18,23]. This additionally supports our findings showed a high degree of mutational heterogeneity among the patients studied.

We identified 6 novel mutations across the CHST6 gene in 6 MCD patients as described in the result section. Of these, we have identified a novel missense mutation in patient from the family 9 that leads to the replacement of glutamic acid to glutamine (E274Q) while in an independent studies from different ethnic background (Egypt, Japanese, American) with MCD; they have identified the same missense mutation (E274K) though the glutamic acid was replaced by lysine and the same mutation was identified in a patient from family 28 , but in a heterozygous state suggesting that the second mutation may be present in the deep intronic or regulatory region of CHST6 gene which was not covered by Sanger sequencing $[15,18]$. This mutation was highly conserved across different orthologous species (Figure 2). Polyphene 2, SIFT and Mutation taster also predicted the mutation was pathogenic in nature. In addition, we have used SOPMA tool that also predicted the un-stability (instability index 45.13) in the protein structure. Moreover, additional evidences also supports our findings that the secondary structure of protein was altered by the missense mutation. Which causes protein unstability thus leads to deficient enzyme activity [12].

Interestingly, we have also identified a novel homozygous nonsense mutation (S53X) in a patient from family 36 was highly conserved across different orthologous species. Polyphene 2, SIFT and Mutation taster also predicted the mutation was pathogenic. SOPMA also predicted that this cause changes in protein stability (instability index 58.93) that leads to the formation of unstable protein. We have identified one more novel homozygous nonsense mutation (S81X) was highly conserved with the un-stability (instability index 50.87) in the protein structure that leads to the formation of unstable protein. These nonsense mutations may lead to the absence of proteins due to nonsense-mediated decay (NMD) of the mRNA suggesting that these mutations might be expected to be associated with an early onset and/or severe form of MCD affecting both the eyes [14].

In addition, we have identified one novel homozygous missense mutation (R202H). It was not conserved across the species but Polyphene 2, SIFT and Mutation taster predicted the mutation as pathogenic in nature. Though, this particular change was not conserved. However, its mild secondary structural modifications with instability index 46.24 may be responsible for the loss of enzyme activity.
Apart from the novel mutations described above; we have identified 12 known mutations including a hotspot deletion mutation (Q182RfsX198) in the coding region of the CHST6 gene that causes frameshift changes in the upstream region of CHST6 gene due to nucleotide sequence similarity of CHST5 and CHST6 genes and the adjacent regions or it could be due to chromosomal crossover in CHST5 and CHST6 genes [18]. Previous studies have revealed that frameshift mutations of CHST6 gene may lead to severe MCD phenotypes with much deeper grey white deposits [30].

Additionally, we have also observed a hotspot missense mutation (S53L) in 10 patients from different MCD families that leads to unstable protein (instability index 45.10) this mutation was already reported in 7 patients from seven South Indian families and the same mutation was identified in an American population suggesting a hotspot mutation $[2,12,19]$. Previous studies suggested that this mutation might be present in the 3'-phosphate-binding domain of C-GlcNac-6-ST enzyme. This region of the CHST6 gene contains an active site which might be a mutational hotspot [31].

We failed to identify mutations in 11 patients from 11 different families out of 55 families screened. Warren, et al. also did not identify CHST6 gene mutations in 4 of 51 families screened suggesting that the mutations in these families may be present in a yet to be identified gene or may be present in a deep intronic or promoter region which can be explored by means of extensive linkage analysis [32]. And also this may be asociated with MCD type II phenotype caused by genetic abnormalities in the upstream of CHST6 gene [8].

Taken together, our results indicate the high degree of mutational heterogeneity in Indian population. The coding region of CHST6 gene significantly affected by mutations leads to unstable protein products with altered secondary structure. All the altered amino acid residues are evolutionary conserved among other mammalian species indicating severe functional loss of CHST6 gene. Functional characterization of these novel mutations identified may help to improve the understanding of the disease pathogenesis.

\section{Conclusion}

In conclusion, we identified 6 novels, 8 reported and 2 hotspot mutations in $44 \mathrm{MCD}$ patients from Indian population. Therefore, our screening of CHST6 gene in Indian families with MCD will be useful for genetic diagnosis, carrier detection and genetic counseling to families included in this study and other families with similar disease condition. In addition, our data showed high degree of allelic and locus heterogeneity exists for MCD.

\section{Acknowledgements}

The authors are grateful to the study subjects for their participation in this study. We thank Mr Mohd Hussain Shah for critically reviewing the manuscript and Ms Priya Arumugam for her assistance in data analysis. Finally, we also thank Mrs D. Muthuselvi and Mrs M. Kalarani for their help in sample collection and technical assistance. 


\section{Declarations}

A. Funding This study was supported by Department of Science and Technology-INSPIRE (Grant number- No. DST/ INSPIRE Fellowship/2015/IF150303) New Delhi, India and a research grant (mutt study 1) from Aravind Eye Care System, Madurai, Tamilnadu, India.

B. Conflict of Interest All authors of this manuscript declare that they have no conflict of interest.

C. Ethical approval All procedures performed in studies involving human participants were in accordance with the ethical standards of the Institutional Ethical Committee of the Aravind Eye Care System, Madurai, Tamilnadu, India and with the 1964 Helsinki declaration and its later amendments or comparable ethical standards. Informed consent Informed consent was obtained from all individual participants included in the study.

\section{References}

1. Morgan G. Macular dystrophy of the cornea. The British journal of ophthalmology. 1966; 50(2): 56-57. doi:10.1136/bjo.50.2.57.

2. Warren JF, Aldave AJ, Srinivasan M, Thonar EJ, Kumar AB, Cevallos $\mathrm{V}$, et al. Novel mutations in the CHST6 gene associated with macular corneal dystrophy in southern India. Archives of Ophthalmology. 2003; 121(11): 1608-1612. doi: 10.1001/archopth.121.11.1608.

3. Alzuhairy S, Alkatan HM, Al-Rajhi AA. Prevalence and histopathological characteristics of corneal stromal dystrophies in Saudi Arabia. Middle East African journal of ophthalmology. 2015; 22(2): 179-185. doi: 10.4103/0974-9233.151975.

4. Akova YA, Kirkness CM, McCartney AC, Ficker LA, Rice NS, Steele AD. Recurrent macular corneal dystrophy following penetrating keratoplasty. Eye. 1990; 4(5): 698-705. doi: 10.1038/eye.1990.98

5. Robin AL, Green WR, Lapsa TP, Hoover RE, Kelley JS. Recurrence of macular corneal dystrophy after lamellar keratoplasty. American journal of ophthalmology. 1977; 84(4): 457-461. doi:10.1016/00029394(77)90434-2.

6. Klintworth GK, Reed J, Stainer GA, Binder PS. Recurrence of macular corneal dystrophy within grafts. American journal of ophthalmology. 1983; 95(1): 60-72. doi: 10.1016/0002-9394(83)90334-3.

7. The University of Arizona Health Science, A data base of hereditary ocular diseases. Available at http://disorders.eyes.arizona.edu/ disorders/cornea-dystrophy-macular accessed August 282016.

8. Vance JM, Jonasson F, Lennon F, Sarrica J, Damji KF, Stauffer J et al. Linkage of a gene for macular corneal dystrophy to chromosome 16 . Am J Hum Genet. 1996; 58: 757-762.

9. Jonasson F, Oshima E, Thonar EJ, Smith CF, Johannsson JH, Klintworth GK et al. Macular corneal dystrophy in Iceland. A clinical, genealogic and immunohistochemical study of 28 patients. Ophthalmology. 1996; 103: 1111-1117. doi: 10.1016/S0161-6420(96)30559-9.

10. Liu NP, Baldwin F, Jonasson F, dew-knight S, Stajich J et al. Haplotype analysis in Icelandic families defines a minimal interval for the macular corneal dystrophy type I gene. Am J Hum Genet. 1998; 63: 912-917. doi: 10.1086/302001
11. Funderburgh JL, Funderburgh ML, Mann MM, Conrad GW et al. Physical and biological properties of keratan sulphate proteoglycan. Biochem Soc Trans. 1991; 19: 871-6. doi: 10.1042/bst0190871

12.Sultana A, Mittanamalli SS, Jagannathan A, Balasubramanian D, Kannabiran C, Klintworth GK. Novel mutations of the carbohydrate sulfotransferase-6 (CHST6) gene causing macular corneal dystrophy in India. Molecular vision. 2003; 9: 730-4.

13. Thanh N, Chau HM, Cung LX, Thanh TK, Fujiki K, Murakami A et al. Identification of Novel Mutations of the CHST6 Gene in Vietnamese Families Affected With Macular Corneal Dystrophy in Two Generations. Invest Ophthalmol Vis Sci. 2003; 44: 3310-3316.

14. Birgani SA, Salehi Z, Houshmand M, Mohamadi MJ, Promehr LA et al. Novel mutations of CHST6 in Iranian patients with macular corneal dystrophy. Molecular vision. 2009; 15: 373-377.

15. El-Ashry MF, Abd El-Aziz MM, Shalaby O, Bhattacharya SS et al. Molecular genetic study of Egyptian patients with macular corneal dystrophy. Br J Ophthalmol. 2010; 94: 250-255. doi: 10.1136/ bjo.2009.161810.

16. Huo YN, Yao YF, Yu P. Pathogenic mutations of TGFBI and CHST6 genes in Chinese patients with Avellino, lattice, and macular corneal dystrophies. Journal of Zhejiang University Science B. 2011; 12(9): 687-693. doi: 10.1631/jzus.B1100011.

17. Lee YK, Chang DJ, Chung SK. A Case of Korean Patient with Macular Corneal Dystrophy Associated with Novel Mutation in the CHST6 Gene. Korean J Ophthalmol. 2013; 27(6): 454-458. doi: 10.3341/ kjo.2013.27.6.454.

18. Akama TO, Nishida K, Nakayama J, Watanabe H, Ozaki K, Nakamura $\mathrm{T}$ et al. Macular corneal dystrophy type I and type II are caused by distinct mutations in a new sulphotransferase gene. Nature genetics. 2000; 26(2): 237-241. doi:10.1038/79987.

19. Klintworth GK, Smith CF, Bowling BL. CHST6 mutations in North American subjects with macular corneal dystrophy: a comprehensive molecular genetic review. Mol Vis. 2006; 12:159-176.

20. Funderburgh JL. Mini Review - Keratan sulfate: structure, biosynthesis, and function. Glycobiology. 2000; 10(10): 951-958.

21. Hasegawa N, Torii T, Kato T, Miyajima H, Furuhata A, Nakayasu K et al. Decreased GlcNAc 6-0-sulfotransferase activity in the cornea with macular corneal dystrophy. Investigative ophthalmology \& visual science. 2000; 41(12): 3670-3677.

22. Funderburgh, JL. Keratan sulfate biosynthesis. IUBMB life. 2002; 54(4): 187-194. doi: 10.1080/15216540214932

23. Niel F, Ellies P, Dighiero P, Soria J, Sabbagh C, San C, et al. Truncating mutations in the carbohydrate sulfotransferase 6 gene (CHST6) result in macular corneal dystrophy. Investigative ophthalmology \& visual science. 2003; 44(7): 2949-2953.

24. Yang CJ, SundarRaj N, Eugene JM, Thonar A, Klintworth GK. Immunohistochemical evidence of heterogeneity in macular corneal dystrophy. American journal of ophthalmology. 1988; 106(1): 65-71. doi:10.1016/S0002-9394(14)76390-1

25.Edward DP, Yue BY, Sugar J, Thonar EJ, SunderRaj N, Stock EL et al. Heterogeneity in macular corneal dystrophy. Archives of Ophthalmology. 1988; 106(11): 1579-1583. doi:10.1001/ archopht.1988.01060140747049 
26. Klintworth GK, Oshima E, Al-Rajhi A, Al-Saif A, Thonar EJ, Karcioglu, ZA. Macular corneal dystrophy in Saudi Arabia: a study of 56 cases and recognition of a new phenotype. American journal of ophthalmology. 1997; 124(1): 9-18. doi:10.1016/S0002-9394(14)71637-X

27. Miller SA, Dykes DD, Polesky HF. A simple salting out procedure for extracting DNA from human nucleated cells. Nucleic acids res. 1988; 16: 1215 .

28. Negishi M, Pedersen LG, Petrotchenko E, Shevtsov S, Gorokhov A, Kakuta Y, et al. Structure and function of sulfotransferases. Archives of Biochemistry and Biophysics. 2001; 390(2): 149-157. doi: 10.1006/ abbi.2001.2368.

29. Hassell JR, Newsome DA, Krachner JH, Rodrigues MM. Macular corneal dystrophy: Failure to synthesize a mature keratan sulfate proteoglycan. Proc Natl Acad Sci USA. 1980; 77: 3705-9.
30.Gruenauer-Kloevekorn C, Braeutigam S, Heinritz W, Froster UG. Macular corneal dystrophy: mutational spectrum in German patients, novel mutations and therapeutic options. Graefe's Archive for Clinical and Experimental Ophthalmology. 2008; 246(10): 1441-1447. doi: 10.1007/s00417-008-0836-1.

31. Abbruzzese C, Kuhn U, Molina F, Rama P, De Luca M. Novel mutations in the CHST6 gene causing macular corneal dystrophy. Clinical genetics.2004; 65(2): 120-125. doi: 10.1111/j.00099163.2004.00191.x.

32. Kleinjan DA, Heyningen V. Long-range control of gene expression: emerging mechanisms and disruption in disease. Am J Hum Genet. 2005; 76:8-32. doi: 10.1086/426833. 